\title{
Hierarchical Clustering
}

National Cancer Institute

\section{Source}

National Cancer Institute. Hierarchical Clustering. NCI Thesaurus. Code C63919.

Cluster analysis that uses stepwise partitioning of objects. 